\title{
Study on Variable Step Size SAP Adaptive Filtering Algorithm
}

\author{
Zhicheng Liu, Bo Yang
}

Department of Information, CAPF engineering University, Xi'an, 710086, China

Keywords: adaptive filtering; sub band affine projection (SAP) algorithm; variable step size.

\begin{abstract}
In the interference cancellation system, the SAP (Sub band Affine Projection) algorithm is researched commonly, but the step size is given. In order to solve this problem, this paper puts forward an algorithm that can make the step size change with the variation of output error. The simulation results show that compared to the traditional SAP algorithm, this algorithm have faster rate of convergence, and good steady state error.
\end{abstract}

\section{Introduction}

The step size choice of filter plays an important role in the convergence process of adaptive filtering. The step size not only controls the rate of convergence in the initial stage, but also decides the steady-state performance in the convergence state. The bigger the step size is, the faster the rate of convergence of the algorithm is, and the bigger the steady-state error is.

AP algorithm [1] adopts multiple input vectors to update the weight vector of adaptive filter, which is the pre-whitened influence on the input signal with the adoption of projection of affine space, thus improving the rate of convergence of algorithm. Despite of this, the large calculated amount is the major defect of AP algorithm. As the L stage adaptive filter generally requires the projection matrix formed by $\mathrm{L}$ input vectors to participate in calculation, including matrix inversion and other calculations, this increases the computation complexity of AP algorithm, thus restricting its wide application.

In order to further improve the rate of convergence of algorithm, in the past decade, with the combination of sub band adaptive filter (SAF) [2] and AP algorithm, various SAP algorithms have been proposed, which inherits the common advantages of sub band algorithm and AP algorithm, and avoids matrix inversion through certain methods, or adopts dimensionality reduction to decrease the dimension of projection matrix, thus lowering the computation complexity of algorithm. For example, in the [3] literature, multitier breakdown structure is adopted to make multitier decomposition of the weight vector of adaptive filter. Compared with AP algorithm, this algorithm improves the rate of convergence, and lowers the computation complexity. But the step size should be given.

However, in the [4] literature, Hyun-Chool Shin et al promoted the most representative variable step size sub band affine projection algorithm (VSSAP). This algorithm changed the step size in the iterative process, thus obtaining faster rate of convergence and lower detuning. But it didn't conduct in the sub band. Starting from the steady state error (MSE) thought of decreasing APA algorithm, this paper puts forward a new variable step size sub band affine projection algorithm (VSSAP). The algorithm first divides the sub band. The larger step size is selected in the initial stage of iteration to obtain higher rate of convergence. After the algorithm becomes stable, smaller step size is selected to obtain lower steady state error. Therefore, the algorithm can change step size based on the variation of output error, thus improving the performance of affine projection algorithm [5].

The paper is organized as follows. In the next section, the paper easily propose the SAP algorithm. In Section 3, derive the VSSSAP algorithm with the variation of output error from the formula. Section 4 presents the genetic algorithm steps based on the new algorithm. In Section 5, the computational simulation is made to illustrate the efficiency of the algorithm. Finally, we conclude our paper in section 6. 


\section{Sub band affine projection algorithm}

Sub band affine projection (SAP) algorithm first adopts analysis filter bank to divide the input signal and desired signal into corresponding sub band signals.

$$
\left\{\begin{array}{l}
\mathbf{x}(\mathrm{n})=[\mathrm{x}(\mathrm{n}), \mathrm{x}(\mathrm{n}-1), \cdots, \mathrm{x}(\mathrm{n}-\mathrm{K}+1)]^{\mathrm{T}} \\
\mathrm{x}_{\mathrm{i}}(\mathrm{n})=\mathrm{s}_{\mathrm{i}}^{\mathrm{T}} \mathbf{x}(\mathrm{n}) \\
\mathbf{d}(\mathrm{n})=[\mathrm{d}(\mathrm{n}), \mathrm{d}(\mathrm{n}-1), \cdots, \mathrm{d}(\mathrm{n}-\mathrm{K}+1)]^{\mathrm{T}} \\
\mathrm{d}_{\mathrm{i}}(\mathrm{n})=\mathrm{s}_{\mathrm{i}}^{\mathrm{T}} \mathbf{d}(\mathrm{n}) \\
\boldsymbol{x}_{\mathrm{ij}}(\mathrm{n})=\left[\mathrm{x}_{\mathrm{ij}}(\mathrm{n}), \mathrm{x}_{\mathrm{ij}}(\mathrm{n}-1), \cdots, \mathrm{x}_{\mathrm{ij}}(\mathrm{n}-\mathrm{Ns}+1)\right]^{\mathrm{T}} \\
\mathrm{d}_{\mathrm{i}}(\mathrm{n})=\left[\mathrm{d}_{\mathrm{i}}(\mathrm{n}), \mathrm{d}_{\mathrm{i}}(\mathrm{n}-1), \cdots \cdots, \mathrm{d}_{\mathrm{i}}(\mathrm{n}-\mathrm{Ps}+1)\right]^{\mathrm{T}}
\end{array}\right.
$$

In formula (1), $x(n)$ is the input signal at the time of $n . K$ is the order of analysis filter. Is the weight vector of the number of the analysis filter? $\mathrm{D}(\mathrm{n})$ is the desire signal.

Then SAP make multitier decomposition of sub band signals to form affine projection matrix, and make constant iteration based on the principle of minimal disturbance. Its formula is:

$$
\left\{\begin{array}{c}
X_{\mathrm{ij}}(\mathrm{n})=\left[\boldsymbol{x}_{\mathrm{ij}}(\mathrm{n}), \boldsymbol{x}_{\mathrm{ij}}(\mathrm{n}-1), \cdots, \boldsymbol{x}_{\mathrm{ij}}(\mathrm{n}-\mathrm{Ps}+1)\right] \\
U(n)=\left[\begin{array}{cccc}
X_{00}(\mathrm{n}) & X_{10}(\mathrm{n}) & \cdots & X_{(\mathrm{N}-1)(\mathrm{n})} \\
X_{01}(\mathrm{n}) & X_{11}(\mathrm{n}) & \cdots & X_{(\mathrm{N}-1)(\mathrm{n})} \\
\vdots & \vdots & \ddots & \vdots \\
X_{0(\mathbb{N}-1)(\mathrm{n})} & X_{1(\mathbb{N}-1)(\mathrm{n})} & \cdots & X_{(\mathrm{N}-1)(\mathrm{N}-1)(\mathrm{n})}
\end{array}\right] \\
\mathrm{D}(\mathrm{n})=\left[\mathrm{d}_{0}^{\mathrm{T}}(\mathrm{n}), \mathrm{d}_{1}^{\mathrm{T}}(\mathrm{n}), \cdots \cdots, \mathrm{d}_{\mathrm{N}-1}{ }^{\mathrm{T}}(\mathrm{n})\right]^{\mathrm{T}} \\
E(n)=D(n)-U^{T}(n) \bar{w}_{(n)} \\
\bar{w}(n)=\bar{w}(n)+\mu U^{T}(n)\left[U^{T}(n) U(n)\right]^{-1} E(n)
\end{array}\right.
$$

Wherein, $D(n)$ is the desired signal of sub band; $U^{T}(n)$ is affine projection matrix. $\bar{w}(n)$ Is the coefficient of adaptive filter, and $E(n)$ is the output error in the iteration process. $\mu$ Is the step size.

\section{New variable step size sub band affine projection algorithm (VSSSAP)}

The update matrix formula of Sub band Affine Projection filter coefficient is

$$
\bar{w}(n+1)=\bar{w}(n)+\mu U^{T}(n)\left[U^{T}(n) U(n)\right]^{-1} E(n)
$$

In the formula (3), the following can be obtained when the both sides multiply by data matrix $U(n)$ at the same time

$$
U(n) \bar{w}(n+1)=U(n) \bar{w}(n)+\mu U(n) U^{T}(n)\left[U^{T}(n) U(n)\right]^{-1} E(n)
$$

Define prior uncertainty $e_{a, i}=U(n) \bar{w}(n)$ and posteriori uncertainty $e_{p, i}=U(n) \bar{w}(n+1)$. It can be known from [6] literature that prior uncertainty can be obtained from transposition of $e_{i}=e_{a, i}+v_{i}$.

$$
e_{a, i}=e_{i}-v_{i}
$$

Put formula (5) into formula (4):

$$
e_{p, i}=e_{a, i}-\mu e_{i}=(1-\mu) e_{a, i}-\mu v_{i}
$$

Take norms from the both sides of formula (6), and obtain:

$$
\left\|e_{p, i}\right\|^{2}=(1-\mu)^{2}\left\|e_{a, i}\right\|^{2}+\mu^{2}\left\|v_{i}\right\|^{2}-2 \mu(1-\mu) e_{a, i} v_{i}^{T}
$$

In the iteration process, in order to obtain faster rate of convergence and lower steady state error, it can first minimize posteriori uncertainty, take the derivative of $M$ in formula (3.6), and make it equal to zero, and obtain:

$$
-2(1-\mu)\left\|e_{a, i}\right\|^{2}+2 \mu\left\|v_{i}\right\|^{2}-2 e_{a, i} * v_{i}^{T}+4 \mu e_{a, i} * v_{i}^{T}=0
$$

Then it can acquire:

$$
u=\frac{\left\|e_{a, i}\right\|^{2}+e_{a, i} * v_{i}^{T}}{\left\|e_{a, i}\right\|^{2}+\left\|v_{i}\right\|^{2}+2 e_{a, i} * v_{i}^{T}}
$$


In the actual process, $v_{i}$ is an unknown quantity, and meets with principle of orthogonality with input data $U(n)$. But the variance of $v_{i}$ can be estimated [7-8] and $\left\|e_{i}\right\|^{2}=K \delta_{e, i}{ }^{2}\left\|v_{i}\right\|^{2}=K \delta_{v, i}{ }^{2}$. With the combination of formulas (6) and (9), it can obtain:

$$
u=\frac{\left\|e_{i}\right\|^{2}-\frac{1}{4}\left\|v_{i}\right\|^{2}}{\left\|e_{i}\right\|^{2}}=\frac{\delta_{e, i}{ }^{2}-\frac{1}{4} \delta_{v, i}{ }^{2}}{\delta_{e, i}{ }^{2}}
$$

In the above formula, $\delta_{e, i}{ }^{2}$ is the output error variance in the $\mu_{i}$ moment; $\delta_{v, i}{ }^{2}$ is the random input noise variance; in order to ensure the algorithm convergence, when in $\delta_{e, i}{ }^{2}<\frac{1}{4} \delta_{v, i}{ }^{2}$, stop the upgrading of coefficients.

\section{The process of VSSSAP algorithm}

Through the above formula, we can conclude the algorithm as follows:

Step1 :( Define parameters) $\mathrm{L}=$ tap number; $\mu$ = step factor; $\mathrm{N}=$ sub band number.

$\mathrm{K}=$ the $s_{i}$ order in analysis filter bank $(i=0,1, \cdots, N-1$, which indicates the corresponding sub band number).

Ns $=\mathrm{L} / \mathrm{N}$, which is the multitier decomposition order of filter.

Ps= order of sub band projection.

Step2 :( Initialization) weight coefficient multitier decomposition vector $\bar{w}(0)=0$.

Step3:(Divide sub band) input vector $x(n)=[x(n), x(n-1), \ldots, x(n-K+1)] T$.

Sub band input signal $\mathrm{xi}(\mathrm{n})=\operatorname{siT} \mathrm{x}(\mathrm{n})$ and then take them into extraction.

Input expectation $\mathrm{d}(\mathrm{n})=[\mathrm{d}(\mathrm{n}), \mathrm{d}(\mathrm{n}-1), \ldots, \mathrm{d}(\mathrm{n}-\mathrm{K}+1)] \mathrm{T}$.

Sunbend desired signal di (n) $=\operatorname{siT} d(n)$ and then take them into extraction.

Calculate $\mathrm{n}+1$ moment weight vector estimation:

Step4 : (Affine projection) as for $n=0,1,2 \ldots$

$\boldsymbol{x}_{\mathrm{ij}}(\mathrm{n})=\left[\mathrm{x}_{\mathrm{ij}}(\mathrm{n}), \mathrm{x}_{\mathrm{ij}}(\mathrm{n}-1), \ldots, \mathrm{x}_{\mathrm{ij}}(\mathrm{n}-\mathrm{Ns}+1)\right]^{\mathrm{T}}, \mathrm{x}_{\mathrm{ij}}(\mathrm{n})$ is the signal after extraction $, \mathrm{j}=0,1, \ldots, N-1$, which represents the number of multitier decomposition,

$$
\begin{gathered}
X_{\mathrm{ij}}(\mathrm{n})=\left[\boldsymbol{x}_{\mathrm{ij}}(\mathrm{n}), \boldsymbol{x}_{\mathrm{ij}}(\mathrm{n}-1) \ldots \boldsymbol{x}_{\mathrm{ij}}(\mathrm{n}-\mathrm{Ps}+1)\right] \\
U(n)=\left[\begin{array}{cccc}
X_{00}(\mathrm{n}) & X_{10}(\mathrm{n}) & \cdots & X_{(\mathrm{N}-1) \mathrm{l}}(\mathrm{n}) \\
X_{01}(\mathrm{n}) & X_{11}(\mathrm{n}) & \ldots & X_{(\mathrm{N}-1) 1}(\mathrm{n}) \\
\vdots & \vdots & \ddots & \vdots \\
X_{0(\mathrm{~N}-1)(\mathrm{n})} & X_{1(\mathrm{~N}-1)(\mathrm{n})} & \cdots & X_{(\mathrm{N}-1)(\mathrm{N}-1)(\mathrm{n})}
\end{array}\right] \\
\mathrm{d}_{\mathrm{i}}(\mathrm{n})=\left[\mathrm{d}_{\mathrm{i}}(\mathrm{n}), \mathrm{d}_{\mathrm{i}}(\mathrm{n}-1), \ldots \ldots, \mathrm{d}_{\mathrm{i}}(\mathrm{n}-\mathrm{Ps}+1)\right]^{\mathrm{T}} \\
\mathrm{D}(\mathrm{n})=[\mathrm{d} 0 \mathrm{~T}(\mathrm{n}), \mathrm{d} 1 \mathrm{~T}(\mathrm{n}) \ldots . \mathrm{dN}-1 \mathrm{~T}(\mathrm{n})] \mathrm{T}
\end{gathered}
$$

Step5 :( Variable step size) calculate $n+1$ moment weight vector estimation by the algorithm in this paper.

$$
\begin{gathered}
E(n)=D(n)-U^{T}(n) \bar{w}(n) \\
u_{i}=\left\{\begin{array}{cc}
\frac{\delta_{e, i^{2}}-\frac{1}{4} \delta_{v, i}{ }^{2}}{\delta_{e, i^{2}}} & \delta_{e, i^{2}}>\frac{1}{4} \delta_{v, i}{ }^{2} \\
0 & \text { others }
\end{array}\right. \\
\bar{w}(n+1)=\bar{w}(n)+\mu_{i} U^{T}(n)\left[U^{T}(n) U(n)\right]^{-1} E(n)
\end{gathered}
$$

\section{Computational examples and analysis}

In order to illustrate the feasibility and effectiveness of the algorithm, we use MATLAB toolbox function to simulate two figures. The two figures are both in the same condition that The input adopts the random signal with certain correlation, and in which the sub band number of the algorithm is $\mathrm{N}=2$, sampling number is $4000 \mathrm{~N}=8000$, the order of echo channel identification is 
$\mathrm{L}=32$, and the setting of the order of adaptive filter is the same as that of the order of echo channel. In such condition, the step size factor of SAP is 0.2.Figure 1 shows the convergence speed of the common algorithm in the interference cancellation system, such as NLMS (normalized least mean square algorithm), NSAF(normalized sub band adaptive filter), AP, VSSSAP at the same condition. Figure 2 shows the different step size of SAP algorithm and VSSAP algorithm under the condition of ensuring the minimum MSD after convergence.

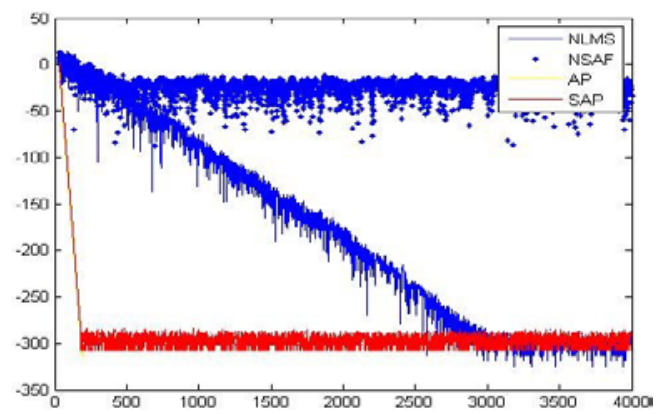

Figure 1 :Convergence Curves of NLMS,NSAF,AP,SAP Algorithm

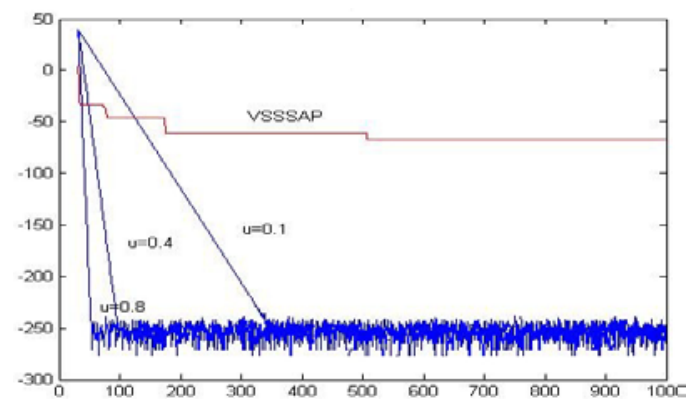

Figure 2 :Convergence Curves of Different Step Size of SAP Algorithm and VSSAP Algorithm

From the figure 1, WO can see that the speed of the SAP algorithm is faster than NLMS and NSAF and it is about the same with AP. In the figure 2, we can see that the algorithm in this paper is the fastest in the different step size. However, because the paper use the output error as the threshold to update step size, the MSD is a little large. But it is also enough for the interference cancellation system.

The amount of calculation of the traditional variable step size affine projection algorithm is much higher than SAP algorithm. The exceeding calculation amount mainly lies in the calculation of step size $\mu_{i}$, if the repeated utilization of $U^{T}(n)\left[U^{T}(n) U(n)\right]^{-1} E(n)$ calculation, and it adds $n+2$ times of multiplication and one division method. With regard to the algorithm proposed in this paper, the major increase of calculation amount is the one division method of calculating step size $\mu_{i}$ and calculating $\delta_{e, i}{ }^{2}$. Under the condition of the output error mean square value as $0, \delta_{e, i}{ }^{2}$ can repeatedly utilize the previous results. The total calculation amount adds two multiplication and one division method.

\section{Conclusions}

Based on that the step size changes with the variation of output error, this paper proposes the variable step size affine projection algorithm. The theoretical analysis and simulation results indicate that although the algorithm put forward in this paper increases the minor computation amount, it has faster rate of convergence and good steady state error, thus obtaining practical value.

\section{References}

[1] K. Ozeki, T. Umeda. An adaptive filtering algorithm using an orthogonal projection to an affine subspace and its properties. Electron. Commun. Jpn., 67-A(5),p.19-27,1984. 
[2] S. S. Pradhan, V. E. Reddy. A new approach to subband adaptive filtering. IEEE Trans. On Signal Processing, 47(3),p.655-664, 1999.

[3] H. Choi, H. D. Bae. Subband affine projection algorithm for acoustic echo cancellation. EURASIP Journal on Advances in Signal Processing, 5,p.909-996, 2007.

[4] Haykin S. Adaptive Filter Theory, 4th Edition. NJ: Prentice-Hall, 2002.

[5] Lee K A, Gan. W S Improving convergence of the NLMS algorithm using constrained subband updates. IEEE Signal Processing Letters,11(9),p.736-739, 2004.

[6] Pradhan S S, Reddy V U. A new approach to subband adaptive filtering. IEEE Tran.Signal Processing, 47(3),p. 655-664, 1998.

[7] Shin H C, Sayed A H,Song W J. Variable Step-Size NLMS and Affine Projection Algorithms, IEEE Signal Processing Letters, 11(2),p.132-135, 2004.

[8] Lee K A, Gan W S, Kuo S M. Mean-square performance analysis of the normalized subband adaptive filter. IEEE Transaction on Signal Processing, 54 (11),p. 4475-4480,2006.

[9] J. D. Gordy, R. A. Goubran. Fast system identification using affine projection and a critically sampled subband adaptive filters. IEEE Trans. on Instrumentation and Measurement,55(4),p.12421249, 2006.

[10] C. Liu, Z. S. He, H. F. Zhang, et al. A variable step size subband affine projection algorithm with dynamic selection of subband filters. IEICE Electronic Express, 8(10),p.715-721, 2011.

[11] M. D. Courville, P. Duhamel. Adaptive filtering in subbands using a weighted criterion.IEEE Trans. on Signal Processing, 46(9),p.2359-2371, 1998.

[12] M. Karthik, A. K. Krishna, K. H. Li, et al. Delay coefficients based variable step size algorithm for subband affine projection adaptive filters. IEICE Electronic Express,6(1),p.20-26,2009. 